\title{
极地冰芯研究的新焦点: NEEM与Dome A
}

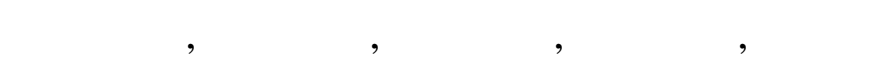 \\ (1) 中国科学院寒区旱区环境与工程研究所冰冻圈科学国家重点实验室, 兰州 730000; \\ (2) 中国极地研究中心, 上海 200129 \\ E-mail: jwren@lzb.ac.cn
}

2008-03-07 收稿, 2008-11-12 接受

中国科学院知识创新工程方向性项目 (编号: KZCX2-SW-354)和国家科技支撑计划(编号: 2006BAB18B01)资助

Ren J W, Xiao C D, Hou S G, et al. New focuses of polar ice-core study: NEEM and Dome A.

Chinese Science Bulletin, 2008, 54(6): 1009—1011, doi: 10.1007/s11434-009-0012-y

摘要 格陵兰冰芯以高分辨率著称, 已经揭示的末次冰期出现的快速气候变化为我们深入了解地球 气候环境变化的规律做出了重要贡献. 目前, 新启动的格陵兰 NEEM 计划以末次间冰期为目标, 试 图更详细地反演末次间冰期及其以来气候环境的变化规律, 为深刻认识与末次间冰期有类似性的现 今气候提供有益的帮助. 南极冰芯以长时间尺度为特色, 对揭示地球轨道尺度的气候变化有独特优 势. 在 Dome A 钻取冰芯以寻求百万年时间尺度的冰芯记录成为南极冰芯研究的焦点.

关键词

冰芯

南极

格陵兰

Dome A

NEEM
冰芯研究是恢复过去气候环境变化记录的重要 手段之一, 其主要特点是时间尺度跨度大、分辨率 高、保真度好，不仅信息量大，而且不同信息可以区 分开来. 极地地区的冰芯记录对长时间尺度气候环 境变化研究尤为重要. 南极内陆由于降水量非常低, 冰芯记录的时间尺度可达几十万年至上百万年, 对 揭示由于地球轨道变化而引起的冰期-间冰期旋回规 律具有独特优势. 北极地区降水量较大, 冰芯记录 以高分辨率见长, 能够辨析气候变化的一些重要细 节. 近半个世纪以来, 格陵兰冰芯记录关于末次冰 期和末次间冰期均存在快速气候变化 ${ }^{[1 \sim 3]}$ 的证据以及 南极冰芯记录给出的 $40 \sim 80$ 万年气候变化总体概貌 [4 6]都已成为古气候变化研究的经典, 对我们深刻认 识地球环境变迁具有划时代意义. 然而, 在全球变 化备受关注的背景下, 极地冰芯研究的脚步并没有 丝毫放慢, 新一轮格陵兰深冰芯计划-NEEM已经启 动, Dome $\mathrm{A}$ 作为南极深冰芯研究的新焦点也已明确.

\section{NEEM 计划背景}

20 世纪 60 至 80 年代在格陵兰冰盖钻取的Camp Century和Dye 3 冰芯其时间尺度就已达到末次冰期,
但因冰芯下部分辨率较低以及冰体运动和底部融化 等原因, 未能很好展现末次冰期气候变化的细节 $\stackrel{[70]}{\text {. }}$ 20 世纪 80 年代末, 欧洲和美国在格陵兰冰盖中部最 高区域同时启动了两个深冰芯计划GRIP(Greenland Ice-core Project)和GISP2(Greenland Ice Sheet Project 2), 力图详细地重建末次间冰期以来的气候变化. 这 两支冰芯钻取地点相距 $30 \mathrm{~km}$, 冰芯长度分别为

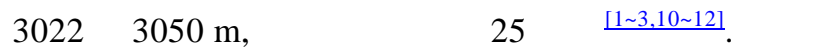
冰芯都无一例外地揭示了末次冰期时频繁的气候突 变特征, 但对末次间冰期是否存在气候突变却存在 争议, 因为冰芯物理特征分析表明, 这段时间的记 录因冰层结构受冰体运动扰动可能存在倒转 ${ }^{[13]}$.

为了验证GRIP和GISP2 冰芯记录, 并确认末次 间冰期是否存在快速气候变化, 20 世纪末在格陵兰 冰盖最高区域偏北的地方实施了 North GRIP (NGRIP). NGRIP冰芯记录分辨率较高, 时间尺度为 12.3 万年, 不仅进一步证实了末次冰期时的快速气 候变化, 而且显示末次间冰期也存在气候突变事件 [14]. 但是, NGRIP冰芯接近底部处于融化状态, 以至 于该冰芯记录末能完全覆盖末次间冰期.

由于末次间冰期和我们目前所处的时代有些相 
象, 而且这一时期的气候变化无疑只是自然因素所 驱动, 研究这一时期的气候变化规律对认识当今气 候变化和格陵兰冰盖的演化非常重要. 特别是目前 对格陵兰冰盖的模拟结果认为 ${ }^{[15]}$, 在未来一个世纪 全球增温幅度为 $1.9 \sim 4.6^{\circ} \mathrm{C}$ 且以后不再发生显著变化 的情景下, 格陵兰冰盖有可能在数百年至上千年间 消融殆尽. 而NGRIP冰芯记录的末次间冰期温度比 现今温度高出 $5^{\circ} \mathrm{C}$, 那时的格陵兰冰盖虽然大量融化 却并未完全消失. 在这种背景下, 新的格陵兰深冰 芯计划又被提出. 该计划主要针对末次间冰期, 而 这一时段又被称作Eemian (对应于海洋同位素 5 e阶 段), 打钻地点定在积累率更高、比NGRIP更靠北的 地方, 所以该计划被命名为NEEM计划.

\section{NEEM 计划的主旨和实施方案}

主要科学目标: 高分辨率重建末次间冰期以来 的气候环境变化, 特别是图解温度、降水、温室气体 等重要指标; 确认末次间冰期时气候是否比现在更 暖; 查验末次间冰期时是否出现快速气候变化; 评 估与末次冰期类似气候条件下的格陵兰冰盖变化; 揭示末次冰期期间暖事件的发生规律; 详细地表述 全新世以来的气候变化; 将末次间冰期和现代气候 变化与预测的全球气候变化情景相关.

实施方案: NEEM 计划几乎联合了世界上所有 开展冰芯研究的科学家参加, 除原来参加 NGRIP 计 划的国家外, 韩国和中国也有兴趣参加。该计划由 丹麦倡导提出, 其他国家参加, 所需经费由各参加国 提供, 主要用于野外工作, 研究经费由各个国家自己 解决. 野外现场工作从 2007 年开始, 2011 年结束.

需要指出的是, NEEM 计划的冰芯钻取地点距 离分冰岭约 $680 \mathrm{~km}$, 如何考虑冰体运动的影响以达 到精确定年至关重要, 因为冰体运动速度从分冰岭 向下游方向是不断增加的.

\section{3 在南极寻求更长时间尺度冰芯记录的重 要意义}

自 20 世纪 60 年代在Byrd站钻取透底冰芯 ${ }^{[16]}$ 以 来, 南极深冰芯记录研究不断取得新的突破. 特别 是Vostok冰芯研究于 20 世纪 80 年代接连给出了 15 和 16 万年气候变化记录 $\frac{17,18]}{2}, 20$ 世纪末又将记录延 伸到 42 万年 ${ }^{[4]}$, 不仅展示了以 10 万年为周期的 4 个
冰期-间冰期旋回, 其 $\mathrm{CO}_{2}$ 记录更成为地球古大气环 境记录的经典. 2004 年EPICA (European Project of Ice Cores in Antarctica) Dome C 冰芯记录则将类似

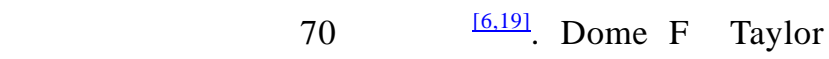
Dome冰芯研究得出的 30 多万年记录也展示了与 Vostok记录极为相似的气候变化特征 ${ }^{[5,20]}$, 在Dome $\mathrm{F}$ 新钻取的一支冰芯其时间尺度估计也在 70 万年左右 [21].

南极冰芯记录时间尺度的不断延长, 使人们看 到了通过南极冰芯记录辨析中更新世气候轨道周期 转变的希望, 因为深海沉积同位素记录显示, 约 100 万年之前受地球轨道变化控制的冰期-间冰期旋回以 4.1 万年为周期 ${ }^{[22]}$. 而EPICA Dome C冰芯记录也显 示, 在距今 40 万年以前, 冰期-间冰期旋回的周期和 幅度似乎都有所减小 ${ }^{[6]}$. 与其他古气候古环境记录 相比, 冰芯记录不仅具有较好的连续性, 更重要的 是可直接提取并分析古大气成分, 这对验证并解释 气候轨道周期转变极为关键. 因此, 在南极地区寻 求时间尺度达到甚或超过 100 万年的冰芯记录具有 特别重要的意义.

\section{Dome A一突破百万年尺度冰芯记录的} 希望之地

Dome A 不仅是南极冰盖的最高点, 也是东南极 大陆的中心, 不仅冰体水平运动很小(这对冰芯定年 极为有利), 其大气环境具有大尺度代表性, 是冰芯 记录研究的理想之地. 但是, 由于这里海拔高、距海 岸远, 被公认为“不可接近地区”, 再加上较早的航空 探测给出的冰体厚度较小, 长期以来并未受到太多 关注.

自 20 世纪末以来的 $10 \mathrm{a}$ 间, 中国南极考察队进 行了 5 次中山站—Dome A断面考察, 2005 年终于 到达Dome A顶点. 对冰体厚度的雷达探测和自动气 象站对表面温度、积累速率的连续监测表明 ${ }^{[23]}$, 该区 域冰体厚度超过 $3000 \mathrm{~m}$, 年平均温度达 $-58^{\circ} \mathrm{C}$, 雪积 累速率约为 $16 \mathrm{~mm}$ 水当量, 是目前已知地球上温度 最低、年雪层厚度最小的地方. 按简单的稳定状态温 度分布和冰体动力学模式推算, $3100 \mathrm{~m}$ 深处冰的年 龄大于 $1.1 \mathrm{Ma}$, 温度为 $-8.4^{\circ} \mathrm{C}$ [24,25]. 因此, Dome A成 为突破百万年尺度冰芯记录的希望之地, 引起国际 科学界极大的兴趣 20,27$]$. 


\section{参考文献}

1 Dansgaard W, Johnsen S J, Clausen H B, et al. Evidence for general instability of past climate from a 250-kyr ice-core record. Nature, 1993, 364: 218-220[DOI]

2 GRIP Project Members. Climate instability during the last interglacial period recorded in the GRIP ice core. Nature, 1993, 364: $203-207 \underline{\text { DOI] }}$

3 Grootes P M, Stuiver M, White J W C, et al. Comparison of oxygen isotope records from the GISP2 and GRIP Greenland ice cores. Nature, 1993, 366: 552-554 $\underline{\text { [DOI] }}$

4 Petit J R, Jouzel J, Raynaud D, et al. Climate and atmospheric history of the past 420000 years from the Vostok ice core, Antarctica. Nature, 1999, 399: 429-436 $\underline{\text { [DOI] }}$

5 Watanabe O, Jouzel J, Johnsen S, et al. Homogeneous climate variability across East Antarctica over the past three glacial cycles. Nature, 2003, 422: 509-512[DOI]

6 EPICA community members. Eight glacial cycles from an Antarctic ice core. Nature, 2004, 429: 623-629[DOI]

7 Dansgaard W, Johnsen S J. A flow model and time scale for the ice core from Camp Century, Greenland. J Glaciol, 1969, 8: 215-223

8 Paterson W S B, Koerner R M, Fisher D A, et al. An oxygen-isotope climatic record from the Devon Island ice cap, Arctic Cananda. Nature, 1977, 266: 508-511 [DOI]

9 Dansgaard W, Clausen H B, Gundestrup N, et al. A new Greenland deep ice core. Science, 1982, 218: 1273-1277[DOI]

10 Ditlevsen P D, Svensmark H, Johnsen S J. Contrasting atmospheric and climate dynamics of the last-glacial and Holocene periods. Nature, 1996, 379: 810-812[DOI]

11 Johnsen S J, Clausen H B, Dansgaard W, et al. The Eem stable isotope record along the GRIP ice core and its interpretation. Quat Res, 1995, 43: 117-124[DOI]

12 Taylor K C, Mayewski P A, Alley R B, et al. The Holocene-Younger Dryas transition recorded at summit, Greenland. Science, 1997, 278: $825-827 \underline{\text { [DOI] }}$

13 Alley R B, Gow A J, Meese D A, et al. Grain-scale processes, folding and stratigraphic disturbance in the GISP2 ice core. J Geophys Res, 1997, 102(C12): 26819-26830[DOI]

14 North Greenland Ice Core Project members. High-resolution record of Northern Hemisphere climate extending into the last interglacial period. Nature, 2004, 431: 147-151묘]

15 IPCC. Climate Change 2007: The Physical Science Basis. Contribution of Working Group I to the Fourth Assessment Report of the Intergovernmental Panel on Climate Change. Cambridge and New York: Cambridge University Press, 2007

16 Robin G de Q. The Climate Record in Polar Ice Sheets. Cambridge: Cambridge University Press, 1983. 196

17 Lorius C, Jouzel J, Ritz C, et al. A 150000-year climatic record from Antarctic ice. Nature, 1985, 316: 591-596[DOI]

18 Jouzel J, Lorius C, Petit J R, et al. Vostok ice core: A continous isotope temperature record over the last climatic cycle (160000 years). Nature, 1987, 329: 403-409[DOI]

19 Masson-Delmotte V, Jouzel J, Parrenin F B, et al. EPICA Dome C: 800000 years of climate and environmental history and relationship with orbital parameters (Abstract). Fourth Mars Polar Science Conference. Houston: Lunar and Planetary Institute, 2006.8108

20 Grootes P M, Steig E J, Stuiver M, et al. The Taylor Dome Antarctic ${ }^{18} \mathrm{O}$ record and globally synchronous changes in climate. Quat Res, 2001, 56: 289-298[DOD]

21 Goto-Azuma K. A $720 \mathrm{kyr}$ ice core chemistry record from Dome Fuji, Antarctica (Abstract). The Ice and Climate Session of IUGG Conference in Perugia, Italy, 9-13 July, 2007

22 Bassinot F C, Labeyrie L D, Vincent E, et al. The astronomical theory of climate and the age of the Brunhes-Matuyama magnetic reversal. Earth Planet Sci Lett, 1994, 126: 91-108[DOI]

23 Xiao C D, Ren J W, Hou S G, et al. Surface characteristics at Dome A (Antarctica) obtained during the first field investigations in 2004/05: A guide to future ice-coring sites. Ann Glaciol, 2008, 48: 82-87 [DOI]

24 侯书贵, 李院生, 效存德, 等. 南极 Dome A 地区的近期积累率. 科学通报, 2007, 52(2): 243-245

25 效存德, 李院生, 侯书贵, 等. 南极冰盖最高点满足钻取最古老冰芯的必要条件: Dome A 最新实测结果. 科学通报, 2007 , 52(20): 2456-2460

26 Nature. China set to drill for Antarctica's oldest ice. Nature, 2006, 444: 255[DOI]

27 Jones N. Polar research: Buried treasure. Nature, 2007, 446: 126-128[DOI] 